\title{
Memory coherence of a sympathetically cooled trapped-ion qubit
}

\author{
J. P. Home, M. J. McDonnell, D. J. Szwer, B. C. Keitch, D. M. Lucas, D. N. Stacey, and A. M. Steane \\ Department of Physics, Clarendon Laboratory, University of Oxford, Parks Road, Oxford OX1 3PU, United Kingdom
}

(Received 19 November 2008; published 22 May 2009)

\begin{abstract}
We demonstrate sympathetic cooling of a ${ }^{43} \mathrm{Ca}^{+}$trapped-ion "memory" qubit by a ${ }^{40} \mathrm{Ca}^{+}$"coolant" ion sufficiently near the ground state of motion for fault-tolerant quantum logic, while maintaining coherence of the qubit. This is an essential ingredient in trapped-ion quantum computers. The isotope shifts are sufficient to suppress decoherence and phase shifts of the memory qubit due to the cooling light which illuminates both ions. We measure the qubit coherence during ten cycles of sideband cooling, finding a coherence loss of $3.3 \%$ per cooling cycle. The natural limit of the method is $O\left(10^{-4}\right)$ infidelity per cooling cycle.
\end{abstract}

DOI: 10.1103/PhysRevA.79.050305

PACS number(s): 03.67.Pp, 37.10.Rs

Trapped ions have been shown to have much promise for quantum information processing (QIP). Multiqubit quantum logic gates [1-4], high-fidelity operations [5,6], and elementary algorithms [7-10] have all been demonstrated. Scaling up from these small scale demonstrations to algorithms involving large numbers of gates, measurements, and individual manipulations of a large number of ions is a major challenge [11]. One of the principal barriers to implementing large-scale quantum information processing with trapped ions is ambient heating of ions caused by fluctuations in the electric potential at the ion. This has been widely observed in ion traps [12-14]. In addition, many proposals involve shuttling, separation, and recombination of ion strings, which introduces heating $[15,16]$. All logic gate schemes demonstrated thus far require the ions to be well within the LambDicke regime for high-fidelity operation [3,17]. Thus for a quantum processor involving ions in trap arrays, the ability to cool ions near the ground state of motion while preserving the logical information stored in them is essential.

One approach to this problem is to cool sympathetically the qubit ion, making use of its Coulomb interaction with another "coolant" ion stored in the same trap [18,19]. Owing to the Coulomb interaction, the normal modes of motion of the ions are shared; therefore by addressing laser cooling only to the "coolant" ion we also cool the logical qubit ion. In order that the light used for cooling does not decohere the qubit(s) stored in the logic ion(s), it is necessary that it couples only weakly to the internal state of the logic ion. The use of two different ion species confined in the same trap represents a physical system which opens up new possibilities for spectroscopy and production of novel motional states, alongside the applications to quantum information processing [20].

Sympathetic cooling of trapped ions near the motional ground state has been reported in crystals of ${ }^{40} \mathrm{Ca}^{+}-{ }^{40} \mathrm{Ca}^{+}$ $[21],{ }^{24} \mathrm{Mg}^{+}-{ }^{9} \mathrm{Be}^{+}[22]$, and ${ }^{27} \mathrm{Al}^{+}-{ }^{9} \mathrm{Be}^{+}[20]$. In this Rapid Communication, we report near-ground-state sympathetic cooling to a temperature sufficient for fault-tolerant logic gates to be possible, using a pair of isotopes ${ }^{40} \mathrm{Ca}^{+}-{ }^{43} \mathrm{Ca}^{+}$, and we directly measure the coherence of the qubit ion while the cooling proceeds [23]. By a detailed analysis of the sources of decoherence due to the sympathetic cooling, we show that infidelity $O\left(10^{-4}\right)$ per cooling cycle is possible in principle.
Consider a crystal of two ions: one coolant and one logic ion, the latter storing a qubit in its internal state. The goal is to cool one or more normal modes of motion from some initial state $\rho_{i}$ to a cold final state $\rho_{f}$, without decohering the qubit. Generally speaking, some form of resolved sideband cooling is needed and pulsed cooling is usually preferable because it is more readily optimized. However, every process has some decohering effect. We quantify this by a parameter $\epsilon$ defined as the drop in interference fringe contrast observed in a Ramsey-type interference experiment on the qubit, per cycle of pulsed sideband cooling of the ion pair.

In quantum computing, one aims to suppress the infidelity per quantum logic gate below some required level $\gamma$. Values in the range $10^{-5}<\gamma<10^{-3}$ are typically discussed [11]. Assuming the logic gate is insensitive to the prepared motional state to lowest but not higher orders then the contribution of thermal motion to the gate infidelity is on the order $\gamma_{T} \simeq 0.3 \pi^{2} \eta^{4} \bar{n}(\bar{n}+1)$, where $\eta$ is the Lamb-Dicke parameter and $n$ is the motional quantum number [17]. Since $\eta$ is typically on the order 0.1 , this suggests that $\bar{n}$ must be comfortably below 1 , but it does not need to be extremely small. Let $N$ be the number of cycles of sideband cooling required to achieve this, then $\gamma \geq \gamma_{T}+N \epsilon$. $N$ is set by the gap between the desired motional state $\rho_{f}$ and the state before cooling $\rho_{i}$, and by the ion masses. In a computer, $\rho_{i}$ would be determined by electric field noise and by the precision of the ion transport and splitting/combining operations in a trap array. We assume it would be roughly thermal, with a mean excitation on the order 1 . In our experiments, we study the process in the relevant parameter regime by producing such conditions in a single trap. Values in the region $1 \leq N<10$ are needed, and therefore the goal is $\epsilon \lessgtr 10^{-4}$. This requires selective addressing, i.e., driving the coolant ion through the cooling cycle, without exciting the internal degrees of freedom of the logic ion. $\epsilon$ is a function primarily of the ratio $R$ of excitation rates of the two ions by the applied laser beam(s). In pulsed sideband cooling, $R$ is the number of photons scattered by the logic ion per cooling cycle.

Two approaches to attaining small $R$ have been discussed. The first makes use of a tightly focused laser beam to address one ion only [21]. Assuming Gaussian optics and the same atomic coefficients for neighboring ions, one has $R \geq \exp \left(-2 s^{2} / w^{2}\right)$ where $s$ is the ion separation and $w$ is the beam waist. This approach involves very demanding techni- 


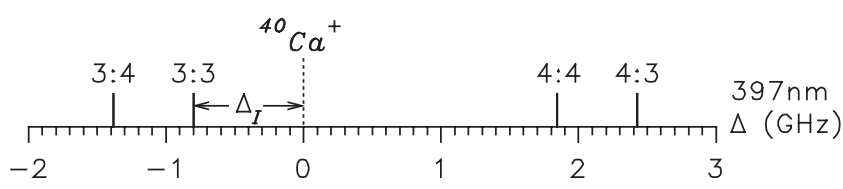

FIG. 1. Frequencies at zero magnetic field of the four hyperfine components of the $S_{1 / 2} \rightarrow P_{1 / 2}$ transition in ${ }^{43} \mathrm{Ca}^{+}$, relative to the same transition in ${ }^{40} \mathrm{Ca}^{+}$. The labels give $F_{S}: F_{P}$, the total angularmomentum quantum number for the $S(P)$ level. $\Delta_{I}$ in Eq. (1) is taken as the smallest isotope shift in the set; the influence of the other components is included via the factor $h$.

cal constraints which become greater at higher trap frequencies, since the ions are closer together. It does however allow the logic and coolant ions to be the same species, which has the advantage that the same laser sources can be used for both the cooling and the control of the logic ion.

The second approach is to use two different ion species. Here the cooling light couples weakly to the logic ion because it is far (many $\mathrm{nm}$ ) from resonance with transitions in this ion. This approach has been used with a ${ }^{24} \mathrm{Mg}^{+}-{ }^{9} \mathrm{Be}^{+}$ crystal to achieve ground-state cooling [22].

We adopt a variant of the second approach, using two different isotopes of the same element. This has previously been used to Doppler cool ions [24]. There are some advantages to having the two ions of similar mass [18], and through the use of electro-optic modulators (EOMs) the same laser sources can be used for the control of both isotopes. However, off-resonant coupling of cooling light to the logic ion is stronger than for the case of two different elements.

For the laser detunings used in our pulsed sideband cooling experiments, $R$ is given approximately by

$$
R=R_{\mathrm{rsb}}+R_{\sigma} \simeq g \frac{\pi \Gamma}{\eta \Delta}+h\left(\frac{\Gamma}{2 \Delta_{I}}\right)^{2},
$$

where $\Delta$ is the detuning of the Raman sideband cooling laser from the ${ }^{40} \mathrm{Ca}^{+} 4 S_{1 / 2} \rightarrow 4 P_{1 / 2}$ transition, $\Gamma$ is the decay rate of the $P_{1 / 2}$ level, $\Delta_{I}$ is the isotope shift (see Fig. 1 ), and $g, h$ are factors of order unity which take account of the angularmomentum coefficients and the sum over all the hyperfine components.

The two terms represent scattering during the redsideband transition and during the repump pulse, respectively. Not all photon scattering leads to decoherence of the qubit [25]. However, for the parameter values of our experiments, the difference between the total scattering rate and the inelastic-scattering rate is small (we return to this point below). The resulting decoherence from both sources is $\simeq R$.

In addition to photon scattering, which irreversibly decoheres the qubit, the cooling beams also differentially light shift the qubit levels, resulting in a $\hat{\sigma}_{z}$ rotation, and they drive various off-resonant Raman processes in the logic ion. The light shift is well defined and can be taken into account or compensated in a practical computer. The Raman processes are hard to keep track of and therefore contribute to $\epsilon$; they can be kept small by a judicious choice of magnetic field and laser polarization and by reducing the laser intensities.

In our experiments, the logical qubit is stored in the ${ }^{43} \mathrm{Ca}^{+} \quad$ "clock" states $|\downarrow\rangle \equiv\left|S_{1 / 2}, F=4, M_{F}=0\right\rangle \quad$ and $|\uparrow\rangle \equiv\left|S_{1 / 2}, F=3, M_{F}=0\right\rangle$. We have observed long coherence times for these states [14], making this a good candidate for QIP. The ${ }^{40} \mathrm{Ca}^{+}$ion is the coolant, cooled by Doppler and Raman cooling via the $397 \mathrm{~nm} 4 S_{1 / 2} \rightarrow 4 P_{1 / 2}$ transition, hereafter designated $S P$, with repump light on $3 D_{3 / 2} \rightarrow 4 P_{1 / 2}$. The $S P$ frequencies of ${ }^{43} \mathrm{Ca}^{+}$relative to ${ }^{40} \mathrm{Ca}^{+}$are given in Fig. 1 .

The ions are stored in a linear Paul trap largely as described in [26]. The secular frequencies for a single trapped ${ }^{40} \mathrm{Ca}^{+}$ion are $\left\{\omega_{r}, \omega_{z}\right\} \simeq 2 \pi \times\{700,500\} \mathrm{kHz}$ for radial and axial motion, respectively. A two-ion crystal of ${ }^{40} \mathrm{Ca}^{+}-{ }^{43} \mathrm{Ca}^{+}$exhibit radial oscillation near $\omega_{r}$ and two modes of axial motion. In the lower (higher) -frequency axial mode, the oscillation of the two ions is nearly in phase (out of phase). The axial mode frequencies are $\omega_{\text {in }}=0.98 \omega_{z}$ and $\omega_{\text {out }}=1.70 \omega_{z}$.

A $0.17 \mathrm{mT}$ magnetic field splits the two ground states of ${ }^{40} \mathrm{Ca}^{+}$by $\omega_{0} \simeq 2 \pi \times 4.8 \mathrm{MHz}$ and removes the Zeeman degeneracy of ${ }^{43} \mathrm{Ca}^{+}$. Fluorescence from each ion is observed by turning on its respective Doppler cooling beams (involving near resonant 397 and $866 \mathrm{~nm}$ light). The ${ }^{43} \mathrm{Ca}^{+} 397 \mathrm{~nm}$ beam is tuned to the $F=4 \rightarrow 4$ hyperfine component of $S P$ and an EOM at $3.220 \mathrm{GHz}$ provides a sideband to repump on $F=3 \rightarrow 4$.

Three stages of cooling (of ${ }^{40} \mathrm{Ca}^{+}$) are required to reach the ground state of motion. The first is Doppler cooling using the cooling beams, with the $397 \mathrm{~nm}$ light detuned half a linewidth below SP. The second stage is continuous Raman sideband cooling using two beams derived from the $397 \mathrm{~nm}$ laser detuned $130 \mathrm{MHz}$ below SP. The final stage is the pulsed Raman sideband cooling. In each cooling cycle, first a Raman red-sideband $\pi$ pulse transfers population from $S_{1 / 2}$ $\left|M_{J}=-1 / 2, n\right\rangle \rightarrow\left|M_{J}=+1 / 2, n-1\right\rangle$ (mean duration $15 \mu \mathrm{s}$ ) then a $\tau_{\sigma}=10 \mu$ s repump pulse, resonant with $S P$ and polarized $\sigma^{-}$, is used to optically pump from $M_{J}=+1 / 2$ to $M_{J}=-1 / 2$ via $P_{1 / 2}$.

The Raman sideband pulses are implemented using two beams derived from the same laser detuned by $\Delta=2 \pi \times 30 \mathrm{GHz}$ above $S P$ and with a frequency difference $\delta$ introduced by acousto-optic modulators. One beam is directed along the magnetic field and is vertically polarized; the other is at $60^{\circ}$ to the magnetic field and is horizontally polarized. The difference wave vector is along the axis of the trap. The repump beam is directed along the magnetic field.

After cooling both axial modes, motional temperatures were inferred from sideband observations [27]. With a fixedlength probe pulse, $\delta$ is scanned and the excitation probability detected (see Fig. 2). For a thermal state in the LambDicke regime, the ratio $r$ of amplitudes of the red and blue sidebands gives the mean vibrational quantum number for the mode: $\bar{n}=r /(1-r)$. For the data shown, we find $\bar{n}_{\text {in }}=0.06(3)$ and $\bar{n}_{\text {out }}=0.07(5)$ for the in- and out-of-phase modes, respectively. For a gate, this would give $\gamma_{T} \lesssim 1.2 \times 10^{-4}$.

Next we describe the experimental observation of qubit coherence during sympathetic sideband cooling of the outof-phase mode. The results extend readily to cooling mul- 

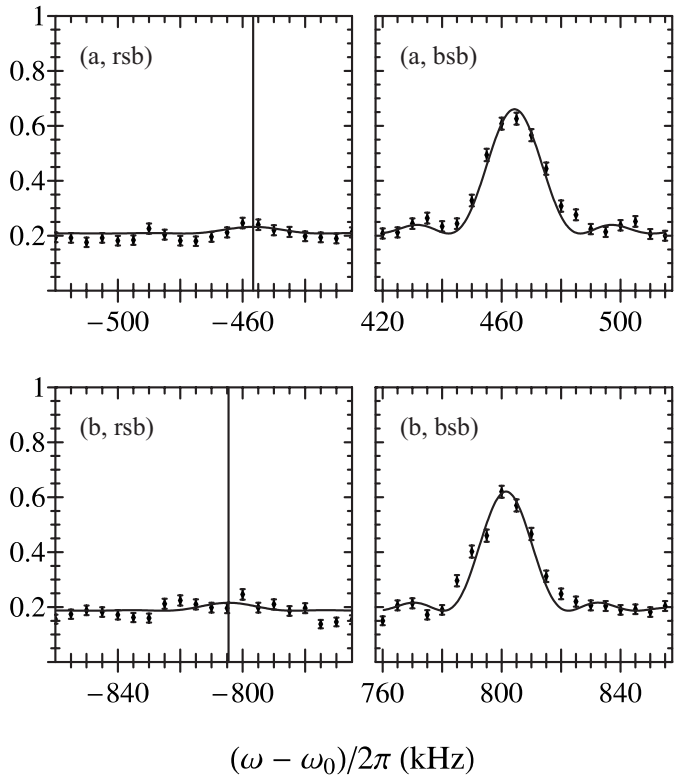

FIG. 2. Results of scanning a 24- $\mu$ s-Raman probe pulse over the red (rsb) and blue (bsb) sidebands of (a) the in-phase mode and (b) the out-of-phase mode, after cooling. The data show the fraction of times in 500 repetitions that no ${ }^{40} \mathrm{Ca}^{+}$fluorescence was observed after a detection pulse. It is fitted with sinc functions, and from the ratio of the fitted amplitudes we obtain $\bar{n}_{\text {in }}=0.06(3)$ and $\bar{n}_{\text {out }}$ $=0.07(5)$. Vertical lines indicate the (known) frequencies of the red sidebands.

tiple modes. We measure the mode temperature as above, and we measure the qubit coherence by Ramsey interferometry. A given experimental sequence consists of precooling to $\bar{n} \sim 0.6$ (of the order to be expected after controlled transport in an ion trap computer) then a standard Ramsey sequence, with one or more sideband cooling cycles inserted in the gap between the Ramsey $\pi / 2$ pulses, followed by the detection of either the qubit state or the motional state. One complete measurement of the pair $\left(\bar{n}_{\text {out }}, \epsilon\right)$ involves 40 data points (20 each for scans of final sideband strength and Ramsey frequency), where each data point is obtained from 500 repetitions of a given experimental sequence. The Ramsey fringes are fitted with a sine function and compared to a control experiment with no sideband cooling.

Qubit state preparation is by turning off the EOM in the $397 \mathrm{~nm}$ beam. This results in optical pumping to $S_{1 / 2} F=3$, with near uniform filling of the Zeeman sublevels, giving $15(1) \%$ preparation of $|\uparrow\rangle$ (an additional $\pi$-polarized beam would increase this preparation fidelity to $\sim 99 \%$ [28]). Resonant Rabi flopping of the qubit is driven by $3.226 \mathrm{GHz}$ microwaves applied to one of the trap electrodes. Readout is accomplished by first using a short pulse of circularly polarized $393 \mathrm{~nm}$ light to selectively transfer population from $F=4$ into the $D_{5 / 2}$ "shelf," with probabilities $0.90(1)$ and 0.002 (1) for $|\downarrow\rangle$ and $|\uparrow\rangle$, respectively [6]. The Doppler cooling lasers are then turned on, and the presence of fluorescence indicates whether the ion was shelved.

Our results are shown in Fig. 3. Mean vibrational quantum number and Ramsey fringe amplitude are shown as a function of $N$ for two sets of data. The fringe amplitude was
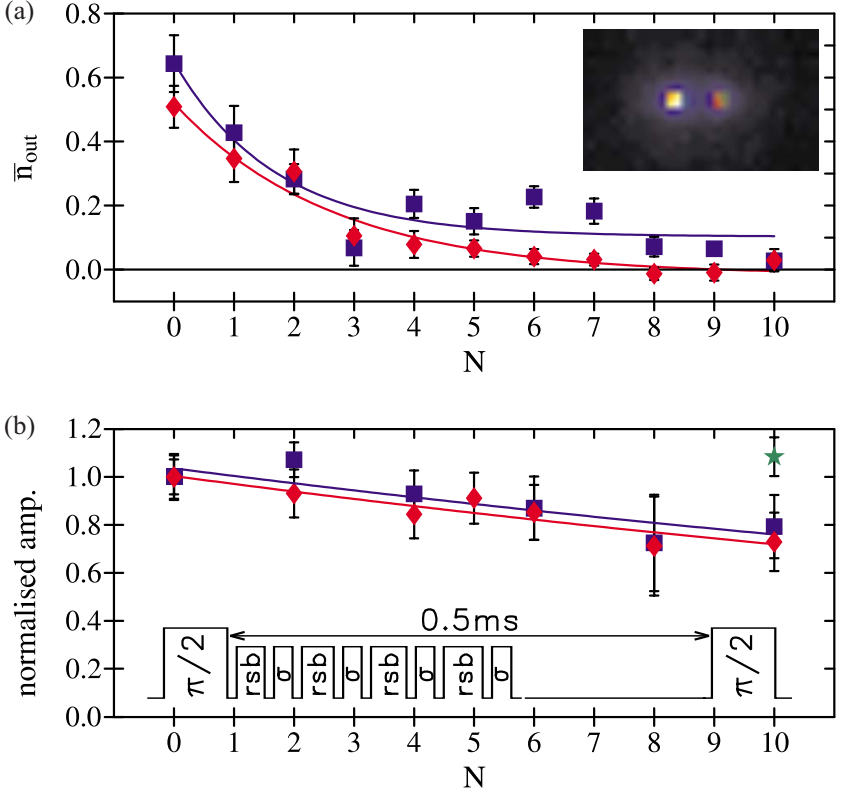

FIG. 3. (Color online) (a) $\bar{n}_{\text {out }}$ as a function of the number of cycles $N$ of pulsed Raman sideband cooling. Each point is obtained from data similar to that shown in Fig. 2. (b) Ramsey fringe amplitude normalized to a control experiment with no sideband cooling cycles. Two data sets are shown $(\boldsymbol{\square}, \downarrow)$; the results are fitted with exponential decay curves. Insets: (a) two-ion fluorescence image and (b) pulse sequence. Also shown is a single point $(\star)$ from an experiment in which only the ten repump $\sigma$ pulses were applied. The amplitude of this fringe pattern relative to the control experiment is $1.08(9)$.

fitted with an exponential function with floated decay constant, giving a loss of coherence per cooling cycle $\epsilon=0.033(4)$. Half of this error is due to scattering [Eq. (1)]; the other half agrees with the estimated contribution of offresonant Raman processes driven by the cooling beams.

Much lower values of $\epsilon$ can be reached. Our experimental parameter values were such that $R$ is dominated by photon scattering during the sideband pulse $R_{\text {rsb }}$. We performed two further experimental tests to establish this and to explore what the natural limits of the method are.

Figure 3(b) shows a further data point from an experiment performed with only the ten repump pulses in the gap between the $\pi / 2$ pulses. From this we calculate an upper bound $\epsilon<7 \times 10^{-3}$ (with no cooling) at $95 \%$ confidence level. In the second test, a single pulse from the repump laser was turned on in the $5 \mathrm{~ms}$ gap of a Ramsey experiment. The observed population of the $F=4$ hyperfine level as a function of the repump pulse duration is shown in Fig. 4. The scan shows three major effects, for which values are obtained by fitting

$$
P(F=4)=\left(1-e^{-\alpha t}\right)+A\left[1+e^{-\beta t} \cos \left(\Delta_{q} t\right)\right] .
$$

These are a rising base line at rate $\alpha=21(3) \mathrm{s}^{-1}$ due to optical pumping from the "spectator" $F=3, M_{F} \neq 0$ states to $F=4$, oscillations of amplitude $A=0.076(8)$ and frequency $\Delta_{q}=2 \pi \times 623(7) \mathrm{Hz}$ due to the differential light shift of the qubit levels, and decaying amplitude of these oscillations at 


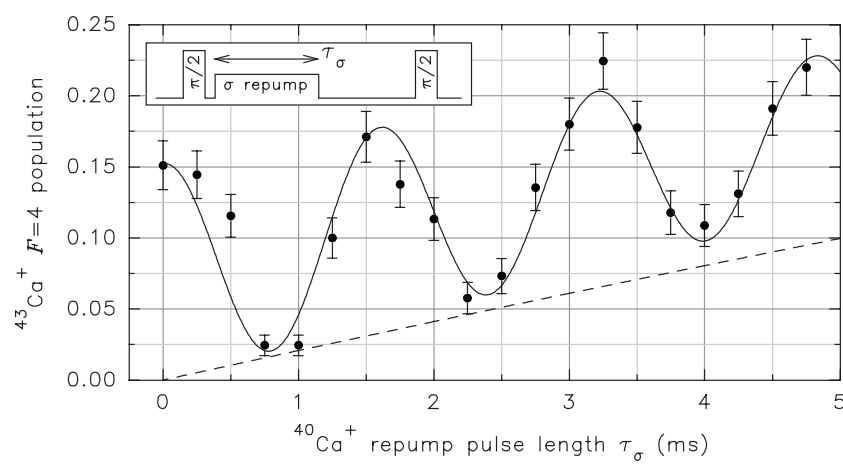

FIG. 4. The population of the ${ }^{43} \mathrm{Ca}^{+} S_{1 / 2}^{F=4}$ level as a function of the duration of a ${ }^{40} \mathrm{Ca}^{+}$repump pulse inserted between two $\pi / 2$ pulses on the "clock" qubit transition. The initial population of $S_{1 / 2}^{F=4, M_{F}=0}$ is $\simeq 0.15$.

$\beta=60(50) \mathrm{s}^{-1} . A$ indicates that $15 \%$ of the population was prepared in the clock state. $\Delta_{q}$ does not decohere the qubit, but it implies a phase shift of $39 \mathrm{mrad}$ per $10 \mu$ s repump pulse which must be taken into account. From $\Delta_{q}$, we infer that the laser intensity was $10.5(1) \mathrm{W} / \mathrm{m}^{2}$. Putting this into a rate equation model of the optical pumping gave an expected transfer to $F=4$ in agreement with the observed base line slope $\alpha$. The decaying amplitude of the oscillations represents decoherence but it is only just observable; the data suggest an upper bound $\beta<150 \mathrm{~s}^{-1}$ and hence $\epsilon=\beta \tau_{\sigma}<1.5 \times 10^{-3}$. The base line slope $\alpha$ directly measures the $F=3 \rightarrow 4$ pumping rate for approximately uniform filling of the Zeeman sublevels of $S_{1 / 2}, F=3$. The scattering $R_{\sigma}$ for an ion $100 \%$ in the clock manifold $(|\uparrow\rangle,|\downarrow\rangle)$, the second term in Eq. (1), can be inferred from this using the known relative component strengths. We obtain $R_{\sigma} \simeq 2 \alpha \tau_{\sigma}=4(1) \times 10^{-4}$. This value is our best estimate of the contribution to $\epsilon$ from the repump process in our cooling experiments.

To conclude, we have observed sympathetic sideband cooling to the ground state of motion $(>90 \%$ occupancy for two modes) and directly demonstrated that laser cooling of a trapped-ion quantum register can be implemented in between coherent qubit rotations with small (3\%) cost in fidelity. Smaller error rates are in principle available by increasing the power and detuning of the Raman sideband cooling laser: this reduces the first term in Eq. (1). Eventually one reaches the regime where Rayleigh elastic scattering dominates, and for our chosen transition in $\mathrm{Ca}^{+}$the contribution to $\epsilon$ is $O\left(10^{-4}\right)$ [25]. The second term in Eq. (1) is calculated to be $1 \times 10^{-4}$ when three photons are scattered from the ${ }^{40} \mathrm{Ca}^{+}$ ion. Extraneous Raman processes in the logic ion can also be reduced to this level by using pure $\sigma$ - and $\pi$-polarized cooling beams and a sideband Rabi frequency smaller by a factor 2. Supposing one coolant ion is used to cool two logic ions, one finds the error from Raman scattering associated with one cooling pulse is similar to that associated with one twoqubit logic gate pulse. To achieve even lower error rates, one could use a transition with smaller width and larger isotope shifts, such as to a metastable $D$ state in $\mathrm{Ca}^{+}$, either for cooling or for logic gates or both $[5,21]$.

This work was supported by IARPA (Contract No. W911NF-05-1-0297), the EPSRC (QIP IRC), the European Commission (FET networks CONQUEST, SCALA), and the Royal Society.
[1] D. Leibfried et al., Nature (London) 438, 639 (2005).

[2] H. Häffner et al., Nature (London) 438, 643 (2005).

[3] D. Leibfried et al., Nature (London) 422, 412 (2003).

[4] F. Schmidt-Kaler et al., Nature (London) 422, 408 (2003).

[5] J. Benhelm et al., Nat. Phys. 4, 463 (2008).

[6] A. H. Myerson et al., Phys. Rev. Lett. 100, 200502 (2008).

[7] M. D. Barrett et al., Nature (London) 429, 737 (2004).

[8] M. Riebe et al., Nature (London) 429, 734 (2004).

[9] K. A. Brickman, P. C. Haljan, P. J. Lee, M. Acton, L. Deslauriers, and C. Monroe, Phys. Rev. A 72, 050306(R) (2005).

[10] J. Chiaverini et al., Science 308, 997 (2005).

[11] A. Steane, Quantum Inf. Comput. 7, 3 (2007).

[12] Q. A. Turchette et al., Phys. Rev. A 61, 063418 (2000).

[13] L. Deslauriers et al., Phys. Rev. Lett. 97, 103007 (2006).

[14] D. M. Lucas et al., e-print arXiv:0710.4421.

[15] M. A. Rowe et al., Quantum Inf. Comput. 2, 257 (2002).

[16] R. B. Blakestad et al., Phys. Rev. Lett. 102, 153002 (2009).

[17] A. Sørensen and K. Mølmer, Phys. Rev. A 62, 022311 (2000).
[18] D. Kielpinski et al., Phys. Rev. A 61, 032310 (2000).

[19] G. Morigi and H. Walther, Eur. Phys. J. D 13, 261 (2001).

[20] T. Rosenband et al., Phys. Rev. Lett. 98, 220801 (2007).

[21] H. Rohde et al., J. Opt. B: Quantum Semiclassical Opt. 3, S34 (2001).

[22] M. D. Barrett et al., Phys. Rev. A 68, 042302 (2003).

[23] In Ref. [20] there is an evidence of coherence preservation during sympathetic Doppler cooling near $\bar{n} \simeq 3$, and in Ref. [22] coherence of ${ }^{9} \mathrm{Be}^{+}$is verified, while the light used for cooling to $\bar{n} \simeq 0.2$ and 0.7 is applied.

[24] B. B. Blinov, L. Deslauriers, P. Lee, M. J. Madsen, R. Miller, and C. Monroe, Phys. Rev. A 65, 040304(R) (2002).

[25] R. Ozeri et al., Phys. Rev. A 75, 042329 (2007).

[26] P. Barton et al., Phys. Rev. A 62, 032503 (2000).

[27] F. Diedrich, J. C. Bergquist, W. M. Itano, and D. J. Wineland, Phys. Rev. Lett. 62, 403 (1989).

[28] J. Benhelm, G. Kirchmair, C. F. Roos, and R. Blatt, Phys. Rev. A 77, 062306 (2008). 